\title{
Easily Created Prediction Model Using Automated Artificial Intelligence Framework (Prediction One, Sony Network Communications Inc., Tokyo, Japan) for Subarachnoid Hemorrhage Outcomes Treated by Coiling and Delayed Cerebral Ischemia
}

\author{
Masahito Katsuki ${ }^{1,2}$, Shin Kawamura ${ }^{2}$, Akihito Koh ${ }^{2}$ \\ 1. Department of Neurosurgery, Iwaki City Medical Center, Iwaki, JPN 2. Department of Neurosurgery, Itoigawa \\ General Hospital, Itoigawa, JPN
}

Corresponding author: Masahito Katsuki, ktk1122nigt@gmail.com

\section{Abstract}

\section{Introduction}

Reliable prediction models of subarachnoid hemorrhage (SAH) outcomes and delayed cerebral ischemia (DCI) are needed to decide the treatment strategy. Automated artificial intelligence (AutoAI) is attractive, but there are few reports on AutoAI-based models for SAH functional outcomes and DCI. We herein made models using an AutoAI framework, Prediction One (Sony Network Communications Inc., Tokyo, Japan), and compared it to other previous statistical prediction scores.

\section{Methods}

We used an open dataset of $298 \mathrm{SAH}$ patients, who were with non-severe neurological grade and treated by coiling. Modified Rankin Scale 0-3 at six months was defined as a favorable functional outcome and DCI occurrence as another outcome. We randomly divided them into a 248 -patient training dataset and a 50patient test dataset. Prediction One made the model using training dataset with 5 -fold cross-validation. We evaluated the model using the test dataset and compared the area under the curves (AUCs) of the created models. Those of the modified SAFIRE score and the Fisher computed tomography (CT) scale to predict the outcomes.

\section{Results}

The AUCs of the AutoAI-based models for functional outcome in the training and test dataset were 0.994 and 0.801 , and those for the DCI occurrence were 0.969 and 0.650. AUCs for functional outcome calculated using modified SAFIRE score were 0.844 and 0.892 . Those for the DCI occurrence calculated using the Fisher CT scale were 0.577 and 0.544 .

Review began 06/02/2021 Review ended 06/07/2021 Published 06/16/2021

() Copyright 2021 Katsuki et al. This is an open access article distributed under the terms of the Creative Commons Attribution License CC-BY 4.0., which permits unrestricted use, distribution, and reproduction in any medium, provided the original author and source are credited.

\section{Conclusions}

We easily and quickly made AutoAI-based prediction models. The models' AUCs were not inferior to the previous prediction models despite the easiness.

Categories: Emergency Medicine, Neurology, Neurosurgery

Keywords: automated artificial intelligence (autoai), deep learning (dl), delayed cerebral ischemia (dci), machine learning (ml), subarachnoid hemorrhage (sah)

\section{Introduction}

A reliable prediction model of subarachnoid hemorrhage (SAH) patient outcomes is needed to decide the treatment strategies and save limited medical resources. Previously, many studies statistically tried to make a prediction model for SAH outcomes [1-8] using neurological severity, age, aneurysm location and size, etc. Their area under the curves (AUCs) of the receiver operating curve varied from 0.70 to 0.90 . Gradually, artificial intelligence (AI) has been used to predict the SAH outcomes. Prediction models using random forests had an accuracy of $71 \%$ from 147 patients [9] or AUC of 0.84 from 441 patients [10]. Recently, deep learning is being used, and it produces better prediction models with an AUC around 0.90, even with a small dataset $[11,12]$.

Similarly, predicting delayed cerebral ischemia (DCI) occurrence is essential. If we could know the DCI occurrence, we could treat it more prophylactically and immediately. As the most widely accepted radiological scores, Fisher computed tomography (CT) scale [13] and modified Fisher scale [14] quantify the amount of bleeding to predict DCI occurrence. Other statistically calculated scores [15-17] using additional 
items have been studied, and the AUCs for DCI occurrence are around 0.7 [18]. AI-based prediction models for DCI occurrence had AUCs of around 0.80 [12,19-22]. However, reports on AI-based prediction models for DCI occurrence remain few.

AI will surpass human wisdom, and the technology is gradually used in the neurosurgical fields [9,10,23-31]. However, the majority of medical staff cannot treat AI technology. This is mainly because of the lack of skilled resources to develop a model and time limitations restricting AI application in clinical practice [24]. Recently, automated AI (AutoAI), so-called "the AI by the AI," has been developed. It is easy to handle by amateurs and will solve these problems with less effort and time. It automatically produces the prediction model within a few minutes as long as the dataset is provided. We herein produced prediction models using the amateur-friendly AutoAI framework, Prediction One (Sony Network Communications Inc., Tokyo, Japan) [32] with an open dataset of SAH patients treated by coiling [33] and compared the utility of the AutoAIbased model to modified SAFIRE score regarding functional outcome and Fisher CT scale regarding DCI occurrence.

\section{Materials And Methods Study population}

We used 298 aneurysmal SAH patients' open dataset from Iwaki City Medical Center [33]. All patients were treated by endovascular coiling. The surgical indication and general management of SAH and DCI, described as "symptomatic vasospasm," are described in the original articles [33,34]. The treatment is according to the Japanese Guidelines for the Management of Stroke 2009 [35] and 2015 [36]. Notably, patients classified with Hunt and Kosnik grades I-III were considered eligible to undergo aneurysm treatment, whereas those with Hunt and Kosnik grades IV and V were basically not suitable for such treatment. However, patients younger than 65 years with Hunt and Kosnik grades IV or V were considered as candidates for receiving the treatment because of their relatively young age. Those aged older than 65 years were carefully considered for aneurysm treatment, and we treated them only when the patients' families wanted them to undergo coiling. Therefore, the surgical indication is strictly limited for those with Hunt and Kosnik grade I-III. Rehabilitation of 150 days as maximum and nutritional support were conducted. Prophylaxis and treatment of complications were also ensured. All patients with SAH who had undergone endovascular coiling received 30 mg fasudil three times a day and dual antiplatelet therapy for a month. Intrathecal infusion of 60000 units urokinase for three days after coiling was usually performed to wash out the SAH. Intrathecal, intravenous infusion of nicardipine or intraarterial infusion of fasudil was performed when necessary for treating symptomatic vasospasm. A ventriculoperitoneal shunt was placed when hydrocephalus was observed.

\section{Clinical variables}

We collected 24 variables from the dataset: age, sex, height, weight, Hunt and Kosnik grade, administration of antithrombotic drugs, history of smoking and drinking, hypertension, diabetes mellitus, and dyslipidemia. We also collected albumin, white blood cell count, triglycerides, total cholesterol, low-density lipoprotein cholesterol, glucose, hemoglobin A1c, and potassium levels at admission. As radiological findings, aneurysm location, aneurysm size, Fisher CT scale [13], temporal muscle thickness and area as an indicator of systemic skeletal muscle mass were also collected [34,37-45]. All variables can be known at admission.

\section{Outcomes}

We set two outcomes. 1) Functional outcomes were assessed using the modified Rankin Scale (mRS) at six months. mRS 0-3 was defined as a favorable outcome. 2) We defined "symptomatic vasospasm" in the dataset as another outcome, DCI occurrence, which was diagnosed by computed tomography angiography, magnetic resonance imaging, or magnetic resonance angiography with symptoms [33].

\section{Making the prediction model by Prediction One}

We used Prediction One (version 2.2) to make the prediction model. We divided the 298 patients randomly into a 248-patient training dataset and a 50-patient test dataset. Prediction One read the 248 patients' data, automatically adjusted and optimized the variables in a way that is easy to process statistically and mathematically, and select an appropriate algorithm with ensemble learning. The missing values were automatically compensated. Prediction One made the best prediction model by an artificial neural network with 5-folds cross-validation. The details are trade secrets and could not be provided.

We let the Prediction One software make two prediction models using the 248-patient training dataset using all 24 variables described above. One was to predict functional outcomes, and the other was to predict DCI occurrence. The AUC of each model and stronger variables were automatically calculated. Then, we performed tests using the 50-patient test dataset. We calculated the AUCs of the models for training and test datasets.

\section{Functional outcome prediction using modified SAFIRE score}

As the third model in this study, we investigated modified SAFIRE scores and evaluated its AUCs using the 


\section{Cureus}

same 248-patient training and 50-patient test datasets. Original SAFIRE score [8] consists of four items: age, World Federation of Neurosurgical Societies grade assessed after neurological resuscitation (rWFNS grade) $[8,46]$, aneurysm size, and Fisher CT scale. rWFNS grade is used in the original SAFIRE score, but we acquired Hunt and Kosnik grade. Therefore, we used Hunt and Kosnik grade instead of rWFNS grade and named this scoring as a modified SAFIRE score (Table 1). After calculating the modified SAFIRE score, we investigated the association between the functional outcomes and the raw total modified SAFIRE score ranging from 0 to 22. Its AUCs for the functional outcome were calculated, and we compared them with those from Prediction One's model.

\begin{tabular}{|c|c|}
\hline Variables & Points \\
\hline \multicolumn{2}{|l|}{ Size of the aneurysm } \\
\hline$<10 \mathrm{~mm}$ & 0 \\
\hline $10-19.9 \mathrm{~mm}$ & 2 \\
\hline$>\mathrm{or}=20 \mathrm{~mm}$ & 6 \\
\hline \multicolumn{2}{|l|}{ Age } \\
\hline$<50$ у.о. & 0 \\
\hline $50-60$ у.о. & 1 \\
\hline $60-70$ у.о. & 2 \\
\hline >or 70 y.o. & 5 \\
\hline \multicolumn{2}{|l|}{ Fisher grade } \\
\hline $1-3$ & 0 \\
\hline 4 & 2 \\
\hline \multicolumn{2}{|c|}{ Hunt and Kosnik grade instead of rWFNS grade $\dagger$} \\
\hline I & 0 \\
\hline II & 2 \\
\hline III & 3 \\
\hline IV & 6 \\
\hline V & 9 \\
\hline Total original SAFIRE score $[8]^{*}$ & Risk of mRS 4-6 at 2 months* \\
\hline $0-2$ & $<10 \%$ \\
\hline $3-5$ & $10-25 \%$ \\
\hline $6-8$ & $25-50 \%$ \\
\hline $9-15$ & $50-90 \%$ \\
\hline $15-22$ & $>90 \%$ \\
\hline
\end{tabular}

\section{TABLE 1: Modified SAFIRE score}

mRS; modified Rankin Scale, rWFNS; World Federation of Neurosurgical Societies grade assessed after neurological resuscitation, y.o.; years old, *; In this study, we did not use this probability but the total modified SAFIRE score. We evaluate the association of the functional outcomes and the total modified SAFIRE score ranging from 0 to 22. $\dagger$; Original SAFIRE score uses rWFNS grade. We modified the score using Hunt and Kosnik grade instead of rWFNS grade.

\section{DCl occurrence prediction using Fisher CT scale}

As the fourth model in this study, we investigated the relationship between Fisher CT scale [13] and DCI occurrence using the same 248-patient training and 50-patient test datasets. In Japan, original Fisher CT scale is often used instead of modified Fisher CT scale $[35,36]$. The AUCs for DCI occurrence were calculated, and we compared them with those from Prediction One's model. 


\section{Cureus}

\section{Statistical analysis}

The difference between the training and test data was evaluated by Fisher exact test or Mann-Whitney U test using Statcel 4 (OMS Publishing Inc., Saitama, Japan). We calculated AUCs using Python 3.8.5 on Google Collaboratory with NumPy (version 1.19.5), Pandas (1.1.5), Seaborn (0.11.1), and matplotlib (1.3.1).

\section{Ethics}

This article uses the anonymized open dataset provided by the other hospital from the Data in Brief journal [33], so we did not need any written informed consent directly from the patients for this article. Therefore, the Itoigawa General Hospital Ethics Committee granted a waiver.

\section{Results}

\section{Clinical characteristics}

The 298 SAH patients' data (mean age 63.7 y.o., 208 women and 90 men) were used, and the details are available in the original article [33]. The mean Hunt and Kosnik grade was 2.62, Fisher CT scale 3.16, modified SAFIRE score 6.20, and the modified Rankin Scale at six months 1.89. Two hundred and eighteen patients (73\%) had favorable outcomes, and 57 of the 296 patients (19\%) had DCI. The last two patients could not be evaluated regarding DCI due to early death after surgery, so they were removed in the analysis for predicting the DCI occurrence. There were no significant differences in the variables between the training and test datasets.

\section{Model development and test}

Prediction One produced each prediction model in less than two minutes, and their AUCs are described in Table 2. The AUCs of the AutoAI-based prediction models for functional outcome in the training and test dataset were 0.994 and 0.801 , and those for DCI occurrence were 0.969 and 0.650 .

\begin{tabular}{|l|l|l|}
\hline Model & $\begin{array}{l}\text { AUC* derived from the training dataset } \\
(\mathbf{n = 2 4 8})\end{array}$ & $\begin{array}{l}\text { AUC* derived from the test dataset } \\
(\mathbf{n}=50)\end{array}$ \\
\hline $\begin{array}{l}\text { Prediction-One-produced model for functional } \\
\text { outcome }\end{array}$ & 0.994 & 0.801 \\
\hline $\begin{array}{l}\text { Modified SAFIRE score for functional outcome } \\
\text { Prediction-One-produced model for the DCI }\end{array}$ & 0.844 & 0.892 \\
\hline $\begin{array}{l}\text { occurrence } \\
\text { Fisher CT scale for the DCl occurrence }\end{array}$ & 0.969 & 0.544
\end{tabular}

\section{TABLE 2: Prediction models for functional outcome and the occurrence}

AUC; area under the curve, CT; computed tomography, DCl; delayed cerebral ischemia, *; AUCs were calculated by Python 3.8.5.

The stronger variables of each model are listed in Table 3. In the model for the functional outcome, Fisher CT scale, Hunt and Kosnik grade, temporal muscle area, age, white blood cell count were stronger variables in order. In that for DCI occurrence, blood glucose, age, potassium, triglycerides, and albumin were stronger variables in order. Regarding DCI occurrence, Fisher CT scale and Hunt and Kosnik grade were not important variables compared to other variables. 


\section{Cureus}

\begin{tabular}{|c|c|c|c|c|}
\hline Variables; order of strength & Model for functional outcome & weight & Model for the DCl occurrence & weight \\
\hline 1 & Fisher CT scale & 0.121 & Blood glucose & 0.063 \\
\hline 2 & Hunt and Kosnik grade & 0.108 & Age & 0.060 \\
\hline 3 & Temporal muscle area & 0.107 & Potassium & 0.060 \\
\hline 4 & Age & 0.099 & Triglycerides & 0.060 \\
\hline 5 & White blood cell count & 0.088 & Albumin & 0.059 \\
\hline 6 & Height & 0.085 & Temporal muscle thickness & 0.056 \\
\hline 7 & Temporal muscle thickness & 0.083 & Temporal muscle area & 0.055 \\
\hline 8 & Aneurysm size & 0.082 & Aneurysm size & 0.052 \\
\hline 9 & Triglycerides & 0.078 & Weight & 0.050 \\
\hline 10 & Weight & 0.071 & Height & 0.049 \\
\hline 14 & Albumin & 0.048 & Fisher CT scale & 0.041 \\
\hline 18 & Potassium & 0.039 & Hunt and Kosnik grade & 0.026 \\
\hline
\end{tabular}

TABLE 3: Stronger variables of each model produced by Prediction One

$\mathrm{CT}$; computed tomography, DCl; delayed cerebral ischemia

\section{Comparison to modified SAFIRE score for functional outcome prediction}

We calculated the modified SAFIRE score in both datasets. The AUCs of the modified SAFIRE score for the functional outcome were 0.844 in the training dataset and 0.892 in the test dataset (Table 2).

\section{Comparison to Fisher CT scale for the DCl occurrence}

The AUCs of the Fisher CT scale for the DCI occurrence were 0.578 in the training dataset and 0.544 in the test dataset. They are inferior to Prediction One's model (Table 2).

\section{Discussion}

We made two AutoAI-based prediction models, the functional outcome prediction model and the DCI occurrence prediction model. The AUCs of the AutoAI-based prediction models for functional outcome in the training and test dataset were 0.994 and 0.801 , and those for DCI occurrence were 0.969 and 0.650 . This is one of the few reports using amateur-friendly AutoAI to produce these models. Our study suggested that AutoAI could easily and quickly produce such models in less than two minutes as long as we provide the dataset.

\section{Advantages and limitations of AutoAl}

Statistically making a prediction model or scoring system needs a large number of samples over thousands, so these studies tend to be country-initiated or academic association-initiated research with vigorous laborintensive efforts. However, the larger the sample size, the less detailed information is available, such as comorbidities, use of antithrombotic drugs, or laboratory test data, and the more there are missing data. Also, the treatment strategies vary from hospital to hospital, and patient backgrounds differ depending on countries and regions. Therefore, these prediction models work as the greatest common denominator but not necessarily applicable to the respective hospital.

The performance of our amateur-friendly AutoAI-based model is similar or a bit inferior to these statistically made models. Previous reports on the AutoAI-based functional prediction model for clipped SAH patients [11] and surgically treated intracerebral hemorrhage patients [31] were reported, and they produced good results. Therefore, we attempted to create prediction models similar to the previous reports expecting that we would have got good results with a dataset of patients who were treated by coiling. However, the results were not superior compared to previous scores.

There is a bias in the dataset [33] in our study. The patients were all treated by coiling. Most of the patients 
with Hunt and Kosnik grade IV and V did not undergo surgery and were rarely included in the dataset. It seems difficult to derive generalizable models from this biased dataset. In the patients treated by the coiling, predicting DCI seems difficult compared to those treated by clipping because the previously reported scores to predict DCI were based on the different sizes and characteristics of cohorts [18]. Regarding predicting outcome, those with Hunt and Kosnik grade IV and V tend to have poor outcomes, so they have strong power to improve the prediction accuracy. Furthermore, there is a difference in the outcome ratios, making it mathematically difficult to make better models. Therefore, our AutoAI-based model using small samples could not conquer the modified SAFIRE score produced using over a thousand samples. Also, our results showed the difficulty of predicting the DCI occurrence based on the information at admission. AutoAI is easy and quick, but it could not make highly accurate prediction models with these small biased datasets.

Hyperparameter tuning and data augmentation are ideally needed, but they are difficult for amateur medical personnel.

\section{Recent Al-based prediction models for functional outcome of SAH}

Statistical functional prediction scores have been developed [1-8], so AI-based functional outcome prediction aims to surpass these statistical scores. De Jong produced an AI-based prediction model using only four items [12]. Katsuki reported AutoAI based prediction model even with a small dataset of 100 patients, which contributes to decision making according to each hospital's treatment strategy [11]. Maldaner reported that the AI-based model's accuracy was improved using the secondary complications and disease information [47]. Including other reports [9,10,48], AI-based prediction for functional outcome has become established. Now AI works on the next stage: predicting rupture risk, automated calculation of hemodynamics, automated morphologic analysis to predict rupture, and automated aneurysm diagnosis [49].

\section{Recent Al-based prediction models for DCl}

Ramos used machine learning to make a DCI prediction model using the clinical information, especially radiological features of the aneurysm, and the model had an AUC of 0.74 [20]. Megjhani reported that an hourly risk score for DCI derived from routine vital signs might have the potential to alert clinicians to DCI [21]. De Jong produced an AI-based prediction model using only four items: age, preexisting hypertension, WFNS grade, and modified Fisher scale with an AUC of 0.72 [12]. Park reported an AI-based model with an AUC of 0.77 using many variables, including vital signs and baseline characteristics with minimum redundancy maximum relevance algorithm [22]. Savarraj also reported an AI-based model with an AUC of 0.75 using clinical features [19].

These studies reported that it is now possible to produce AI-based models with AUCs of around 0.75, and that chronological data such as vital signs are important in addition to the patients' characteristics at admission to increase the accuracies. Our results are inferior to these previous reports, but AutoAI suggests that amateur medical personnel can try to keep up with these cutting-edge researches using an AutoAI.

\section{Limitations of this study}

First, we used Hunt and Kosnik grade at admission, but the original SAFIRE score used the WFNS score assessed after neurological resuscitation $[8,46]$ (rWFNS; e.g., cerebral spinal fluid drainage for acute hydrocephalus or evacuation of an intracerebral hematoma). Also, the SAFIRE score predicts two-month outcomes, but our models predict six-month. These are differences, so simply comparing their AUCs requires caution. Second, we used the original Fisher CT scale, which is often used in Japan, but the modified Fisher CT scale [14] is now widely used abroad except for Japan. In addition, other statistically calculated scores [15-17] are also used to predict DCI, so we should compare our model to such recent predicting scores. Third, the prediction model derived from the dataset cannot always be applied to other institutions, and the training and validation dataset must be updated to keep up with advances in medical science and changes in surgical techniques. Fourth, AutoAI produced models easily and quickly, but the neural network architecture by AutoAI is really in the black box for users.

\section{Conclusions}

We easily and quickly made prediction models using the AutoAI framework Prediction One. The accuracies of the prediction models were not so inferior to those of previous statistically calculated prediction models. Even with a small single-center biased dataset, prediction models made by AutoAI might be useful at the institution. AutoAI frameworks are amateur-friendly, so they may be applied to daily clinical practice in the future. The time will come when even amateurs will be able to use AI with ease.

\section{Additional Information}

\section{Disclosures}

Human subjects: Consent was obtained or waived by all participants in this study. Itoigawa General Hospital Ethics Committee issued approval Not Applicable. This article uses the anonymized open dataset provided by the other hospital from the Data in Brief journal, so we did not need any written informed 
consent directly from the patients for this article. Therefore, the Itoigawa General Hospital Ethics Committee granted a waiver. Animal subjects: All authors have confirmed that this study did not involve animal subjects or tissue. Conflicts of interest: In compliance with the ICMJE uniform disclosure form, all authors declare the following: Payment/services info: All authors have declared that no financial support was received from any organization for the submitted work. Financial relationships: All authors have declared that they have no financial relationships at present or within the previous three years with any organizations that might have an interest in the submitted work. Other relationships: All authors have declared that there are no other relationships or activities that could appear to have influenced the submitted work.

\section{Acknowledgements}

Masahito Katsuki and Shin Kawamura are equally contributing authors.

\section{References}

1. Jaja BN, Cusimano MD, Etminan N, et al.: Clinical prediction models for aneurysmal subarachnoid hemorrhage: a systematic review. Neurocrit Care. 2013, 18:143-53. 10.1007/s12028-012-9792-Z

2. Risselada R, Lingsma HF, Bauer-Mehren A, et al.: Prediction of 60 day case-fatality after aneurysmal subarachnoid haemorrhage: results from the International Subarachnoid Aneurysm Trial (ISAT). Eur J Epidemiol. 2010, 25:261-6. 10.1007/s10654-010-9432-x

3. Abulhasan YB, Alabdulraheem N, Simoneau G, Angle MR, Teitelbaum J: Mortality after spontaneous subarachnoid hemorrhage: causality and validation of a prediction model. World Neurosurg. 2018, 112:e799-811. 10.1016/j.wneu.2018.01.160

4. Zeiler FA, Lo BWY, Akoth E, Silvaggio J, Kaufmann AM, Teitelbaum J, West M: Predicting outcome in subarachnoid hemorrhage (SAH) utilizing the Full Outline of UnResponsiveness (FOUR) Score. Neurocrit Care. 2017, 27:381-9. 10.1007/s12028-017-0396-5

5. Hostettler IC, Sebök M, Ambler G, et al.: Validation and optimization of barrow neurological institute score in prediction of adverse events and functional outcome after subarachnoid hemorrhage-Creation of the HATCH (Hemorrhage, Age, Treatment, Clinical State, Hydrocephalus) score. Neurosurgery. 2020, 88:96-105. 10.1093/neuros/nyaa316

6. Jaja BNR, Saposnik G, Lingsma HF, et al.: Development and validation of outcome prediction models for aneurysmal subarachnoid haemorrhage: the SAHIT multinational cohort study. BMJ. 2018, 360:j5745. 10.1136/bmj.j5745

7. Witsch J, Frey HP, Patel S, et al.: Prognostication of long-term outcomes after subarachnoid hemorrhage: the FRESH score. Ann Neurol. 2016, 80:46-58. 10.1002/ana.24675

8. van Donkelaar CE, Bakker NA, Birks J, et al.: Prediction of outcome after aneurysmal subarachnoid hemorrhage. Stroke. 2019, 50:837-44. 10.1161/STROKEAHA.118.023902

9. Rubbert C, Patil KR, Beseoglu K, et al.: Prediction of outcome after aneurysmal subarachnoid haemorrhage using data from patient admission. Eur Radiol. 2018, 28:4949-58. 10.1007/s00330-018-5505-0

10. de Toledo P, Rios PM, Ledezma A, Sanchis A, Alen JF, Lagares A: Predicting the outcome of patients with subarachnoid hemorrhage using machine learning techniques. IEEE Trans Inf Technol Biomed. 2009, 13:794-801. 10.1109/TITB.2009.2020434

11. Katsuki M, Kakizawa Y, Nishikawa A, Yamamoto Y, Uchiyama T: Easily created prediction model using deep learning software (Prediction One, Sony Network Communications Inc.) for subarachnoid hemorrhage outcomes from small dataset at admission. Surg Neurol Int. 2020, 11:374. 10.25259/SNI_636_2020

12. de Jong G, Aquarius R, Sanaan B, Bartels RHMA, Grotenhuis JA, Henssen DJHA, Boogaarts HD: Prediction models in aneurysmal subarachnoid hemorrhage: forecasting clinical outcome with artificial intelligence. Neurosurgery. 2021, 88:E427-34. 10.1093/neuros/nyaa581

13. Fisher CM, Kistler JP, Davis JM: Relation of cerebral vasospasm to subarachnoid hemorrhage visualized by computerized tomographic scanning. Neurosurgery. 1980, 6:1-9. 10.1227/00006123-198001000-00001

14. Frontera JA, Claassen J, Schmidt JM, et al.: Prediction of symptomatic vasospasm after subarachnoid hemorrhage: the modified Fisher scale. Neurosurgery. 2006, 59:21-7. 10.1227/01.NEU.0000218821.34014.1B

15. Ahn SH, Savarraj JP, Pervez M, et al.: The subarachnoid hemorrhage early brain edema score predicts delayed cerebral ischemia and clinical outcomes. Neurosurgery. 2018, 83:137-45. 10.1093/neuros/nyx364

16. Claassen J, Carhuapoma JR, Kreiter KT, Du EY, Connolly ES, Mayer SA: Global cerebral edema after subarachnoid hemorrhage: frequency, predictors, and impact on outcome. Stroke. 2002, 33:1225-32. 10.1161/01.str.0000015624.29071.1f

17. de Oliveira Manoel AL, Jaja BN, Germans MR, et al.: The VASOGRADE: A simple grading scale for prediction of delayed cerebral ischemia after subarachnoid hemorrhage. Stroke. 2015, 46:1826-31. 10.1161/STROKEAHA.115.008728

18. Fang Y, Lu J, Zheng J, et al.: Comparison of aneurysmal subarachnoid hemorrhage grading scores in patients with aneurysm clipping and coiling. Sci Rep. 2020, 10:9199. 10.1038/s41598-020-66160-0

19. Savarraj JPJ, Hergenroeder GW, Zhu L, et al.: Machine learning to predict delayed cerebral ischemia and outcomes in subarachnoid hemorrhage. Neurology. 2021, 96:e553-62. 10.1212/WNL.0000000000011211

20. Ramos LA, van der Steen WE, Sales Barros R, et al.: Machine learning improves prediction of delayed cerebral ischemia in patients with subarachnoid hemorrhage. J Neurointerv Surg. 2019, 11:497-502. 10.1136/neurintsurg-2018-014258

21. Megihani M, Terilli K, Weiss M, et al.: Dynamic detection of delayed cerebral ischemia: a study in 3 centers . Stroke. 2021, 52:1370-9. 10.1161/STROKEAHA.120.032546

22. Park S, Megihani M, Frey HP, et al.: Predicting delayed cerebral ischemia after subarachnoid hemorrhage using physiological time series data. J Clin Monit Comput. 2019, 33:95-105. 10.1007/s10877-018-0132-5

23. van Niftrik CHB, van der Wouden F, Staartjes VE, et al.: Machine learning algorithm identifies patients at 
high risk for early complications after intracranial tumor surgery: registry-based cohort study. Neurosurgery. 2019, 85:E756-64. 10.1093/neuros/nyz145

24. Staartjes VE, Stumpo V, Kernbach JM, et al.: Machine learning in neurosurgery: a global survey . Acta Neurochir (Wien). 2020, 162:3081-9. 10.1007/s00701-020-04532-1

25. Kim HC, Rhim JK, Ahn JH, et al.: Machine learning application for rupture risk assessment in small-sized intracranial aneurysm. J Clin Med. 2019, 8:683. 10.3390/jcm8050683

26. Azimi P, Mohammadi HR, Benzel EC, Shahzadi S, Azhari S: Use of artificial neural networks to decision making in patients with lumbar spinal canal stenosis. J Neurosurg Sci. 2017, 61:603-11. 10.23736/S03905616.16.03078-2

27. Fujita T, Ohashi T, Yamane K, et al.: Relationship between the number of samples and the accuracy of the prediction model for dressing independence using artificial neural networks in stroke patients. Japanese J Compr Rehabil Sci. 2020, 11:28-34. 10.11336/jjcrs.11.28

28. UPenn and Mayo Clinic's Seizure Detection Challenge Detect Seizures in Intracranial EEG Recordings . (2014). Accessed: December 31, 2020: https://www.kaggle.com/c/seizure-detection.

29. Watanabe O, Narita N, Katsuki M, Ishida N, Cai S, Otomo H, Yokota K: Prediction model of deep learning for ambulance transports in Kesennuma city by meteorological data. Open Access Emerg Med. 2021, 13:23-32. 10.2147/OAEM.S293551

30. Katsuki M, Narita N, Matsumori Y, Ishida N, Watanabe O, Cai S, Tominaga T: Preliminary development of a deep learning-based automated primary headache diagnosis model using Japanese natural language processing of medical questionnaire. Surg Neurol Int. 2020, 11:475. 10.25259/SNI_827_2020

31. Katsuki M, Kakizawa Y, Nishikawa A, Yamamoto Y, Uchiyama T: Postsurgical functional outcome prediction model using deep learning framework (Prediction One, Sony Network Communications Inc.) for hypertensive intracerebral hemorrhage. Surg Neurol Int. 2021, 12:203. 10.25259/SNI_222_2021

32. Sony Network Communications: Prediction One. (2020). Accessed: February 29, 2020: https://predictionone.sony.biz/.

33. Katsuki M, Suzuki Y, Kunitoki K, et al.: Temporal muscle thickness and area with various characteristics data of the patients with aneurysmal subarachnoid hemorrhage who underwent endovascular coiling. Data Brief. 2020, 31:105715. 10.1016/j.dib.2020.105715

34. Katsuki M, Suzuki Y, Kunitoki K, et al.: Temporal muscle as an indicator of sarcopenia is independently associated with Hunt and Kosnik grade on admission and the modified Rankin Scale at 6 month of patients with subarachnoid hemorrhage treated by endovascular coiling. World Neurosurg. 2020, 137:e526-34. 10.1016/j.wneu.2020.02.033

35. The Japan Stroke Society: Japanese Guidelines for the Management of Stroke 2009. The Japan Stroke Society Guideline Committee (ed): Kyowa Kikaku, Tokyo; 2009.

36. The Japan Stroke Society: Japanese Guidelines for the Management of Stroke 2015. The Japan Stroke Society Guideline Committee (ed): Kyowa Kikaku, Tokyo; 2015.

37. Katsuki M, Kakizawa Y, Nishikawa A, Yamamoto Y, Uchiyama T: Lower total protein and absence of neuronavigation are novel poor prognostic factors of endoscopic hematoma removal for intracerebral hemorrhage. J Stroke Cerebrovasc Dis. 2020, 29:105050. 10.1016/j.jstrokecerebrovasdis.2020.105050

38. Katsuki M, Kakizawa Y, Nishikawa A, Yamamoto Y, Uchiyama T: Endoscopic hematoma removal of supratentorial intracerebral hemorrhage under local anesthesia reduces operative time compared to craniotomy. Sci Rep. 2020, 10:10389. 10.1038/s41598-020-67456-x

39. Furtner J, Genbrugge E, Gorlia T, et al.: Temporal muscle thickness is an independent prognostic marker in patients with progressive glioblastoma: translational imaging analysis of the EORTC 26101 trial. Neuro Oncol. 2019, 21:1587-94. 10.1093/neuonc/noz131

40. Steindl A, Leitner J, Schwarz M, et al.: Sarcopenia in neurological patients: Standard values for temporal muscle thickness and muscle strength evaluation. J Clin Med. 2020, 9:1272. 10.3390/jcm9051272

41. Furtner J, Berghoff AS, Schöpf V, et al.: Temporal muscle thickness is an independent prognostic marker in melanoma patients with newly diagnosed brain metastases. J Neurooncol. 2018, 140:173-8. 10.1007/s11060018-2948-8

42. Katsuki M, Yamamoto Y, Uchiyama T, Wada N, Kakizawa Y: Clinical characteristics of aneurysmal subarachnoid hemorrhage in the elderly over 75; would temporal muscle be a potential prognostic factor as an indicator of sarcopenia?. Clin Neurol Neurosurg. 2019, 186:105535. 10.1016/j.clineuro.2019.105535

43. Furtner J, Berghoff AS, Albtoush OM, et al.: Survival prediction using temporal muscle thickness measurements on cranial magnetic resonance images in patients with newly diagnosed brain metastases. Eur Radiol. 2017, 27:3167-73. 10.1007/s00330-016-4707-6

44. Onodera H, Mogamiya T, Matsushima S, Sase T, Kawaguchi K, Nakamura H, Sakakibara Y: High protein intake after subarachnoid hemorrhage improves oral intake and temporal muscle volume. Clin Nutr. 2021, 10.1016/j.clnu.2021.01.040

45. Katsuki M, Narita N, Sasaki K, Sato Y, Suzuki Y, Mashiyama S, Tominaga T: Standard values for temporal muscle thickness in the Japanese population who undergo brain check-up by magnetic resonance imaging. Surg Neurol Int. 2021, 12:67. 10.25259/SNI_3_2021

46. van Donkelaar CE, Bakker NA, Veeger NJ, et al.: Prediction of outcome after subarachnoid hemorrhage: timing of clinical assessment. J Neurosurg. 2017, 126:52-9. 10.3171/2016.1.JNS152136

47. Maldaner N, Zeitlberger AM, Sosnova M, et al.: Development of a complication-and treatment-aware prediction model for favorable functional outcome in aneurysmal subarachnoid hemorrhage based on machine learning. Neurosurgery. 2021, 88:E150-7. 10.1093/neuros/nyaa401

48. Liu J, Xiong Y, Zhong M, Yang Y, Guo X, Tan X, Zhao B: Predicting long-term outcomes after poor-grade aneurysmal subarachnoid hemorrhage using decision tree modeling. Neurosurgery. 2020, 87:523-9. 10.1093/neuros/nyaa052

49. Shi Z, Hu B, Schoepf UJ, et al.: Artificial intelligence in the management of intracranial aneurysms: current status and future perspectives. AJNR Am J Neuroradiol. 2020, 41:373-9. 10.3174/ajnr.A6468 